\title{
The relationship between food group intake and frailty in older adults living in Ireland
}

\author{
M.L. O'Connell ${ }^{1}$, T. Coppinger ${ }^{2}$ and A.L. McCarthy ${ }^{1}$ \\ ${ }^{1}$ Department of Biological Sciences, Munster Technological University, Cork, Ireland and \\ ${ }^{2}$ Department of Sport, Leisure and Childhood Studies, Munster Technological University, Cork, Ireland
}

Frailty is a clinical condition characterised by an increased vulnerability to adverse health outcomes due to physiological system decline $^{(1)}$ and has been described as one of the most problematic issues of ageing ${ }^{(2)}$. Good nutrition has provided some evidence of protection against frailty, but little is known about the contribution of individual food groups to this relationship. The aim of this study was to identify any associations between food group intake and frailty in a cohort of Irish older adults.

A cross-sectional study was undertaken among 142 community-dwelling older adults $(\mathrm{n}=81$ female, $\mathrm{n}=61 \mathrm{male}, 74.1 \pm 6.80$ years $)$ who responded to advertisements placed in newsletters and community settings in Cork, Ireland, from March to August, 2019. Health screening sessions took place in community centres and health clinics across Cork, where dietary intake and frailty status were assessed. Frailty was classified according to the Frailty Phenotype, described as $\geq 3$ (or $1-2$ for pre-frailty) of the following features: weakness, exhaustion, low activity, slowness and weight loss. Food group intake was assessed using a validated food frequency questionnaire (FFQ). Food group intake data were transformed and compared between frailty groups using analysis of variance (ANOVA). The relationship between food group intake and frailty was further assessed using Spearman's correlations, followed by partial correlations adjusted for age, body mass index $(\mathrm{kg} / \mathrm{m} 2)$ and total energy intake (kcal/day). Ethical approval was obtained from Cork Institute of Technology Research Ethics Committee in December, 2018.

A total of $17(12.0 \%)$ participants were classified as frail, while $49(34.5 \%)$ were pre-frail. Those who were frailer had a diet that was lower in fruit, vegetables and fish/fish products and higher in sugars, preserves and snacks $(\mathrm{p}<0.05)$. In adjusted analyses, foods which showed significant $(\mathrm{p}<0.05)$ inverse correlations with frailty score in females were fruit $(\mathrm{pr}=-0.296, \mathrm{p}=0.009)$, vegetables $(\mathrm{pr}=-0.294, \mathrm{p}=0.009)$ and fish/fish products $(\mathrm{pr}=-0.360, \mathrm{p}=0.001)$, while intake of sugars/snacks was positively correlated with frailty score $(\mathrm{pr}=0.270, \mathrm{p}=0.017)$. In males, intake of vegetables $(\mathrm{pr}=-0.283, \mathrm{p}=0.031)$ and fish/fish $\mathrm{products}(\mathrm{pr}=-0.366$, $\mathrm{p}=0.005)$ were inversely correlated with frailty score, while intake of fats/oils $(\mathrm{pr}=0.322, \mathrm{p}=0.014)$ showed a positive correlation.

The results of this study suggest that higher intake of fish/fish products and fruit and vegetables may protect against frailty in Irish older adults, while higher intake of sugars, snacks and fats may increase frailty risk. Further research with larger study samples and longitudinal design is needed to confirm these findings.

\section{References}

1. Xue Q (2011) Clin Geriatr Med 27, 1-15.

2. Clegg A, Young J, Iliffe S, et al. (2013) The Lancet 381, 752-762. 